\title{
Open Data Science to fight COVID-19: Winning the 500k XPRIZE Pandemic Response Challenge
}

\author{
Miguel Angel Lozano1(D) (đ) \\ Miguel Rebollo $^{2}$ (D), Kristina Polotskaya ${ }^{3}$ (D), Miguel Angel Garcia-March ${ }^{2}$ (D) \\ J. Alberto Conejero ${ }^{2}$ (D), Francisco Escolano ${ }^{1}$ (D), and Nuria Oliver ${ }^{4}$ (D) \\ 1 University of Alicante, Alicante, Spain \\ malozano@ua.es \\ 2 IUMPA and VRAIN. Universitat Politècnica de València, València. Spain \\ 3 University Miguel Hernández, Elche, Spain \\ 4 ELLIS (European Lab. for Learning and Intelligent Systems) Unit Alicante, Spain
}

\begin{abstract}
In this paper, we describe the deep learning-based COVID-19 cases predictor and the Pareto-optimal Non-Pharmaceutical Intervention (NPI) prescriptor developed by the winning team of the 500k XPRIZE Pandemic Response Challenge, a four-month global competition organized by the XPRIZE Foundation. The competition aimed at developing datadriven AI models to predict COVID-19 infection rates and to prescribe NPI Plans that governments, business leaders and organizations could implement to minimize harm when reopening their economies. In addition to the validation performed by XPRIZE with real data, the winning models were validated in a real-world scenario thanks to an ongoing collaboration with the Valencian Government in Spain. We believe that this experience contributes to the necessary transition to more evidencedriven policy-making, particularly during a pandemic.
\end{abstract}

Keywords: SARS-CoV-2 · Computational Epidemiology · Data Science for Public Health · Recurrent Neural Networks · Non-Pharmaceutical Interventions - Pareto-front optimization

\section{Introduction}

During a pandemic, predicting the number of infections under different circumstances is important to inform public health, health care and emergency system responses. Different approaches to predict the evolution of a pandemic have been proposed in the literature, including traditional compartmental meta-population models -such as SIR or SEIR [12, complex network [18, agent-based individual 9] and purely data-driven time series forecasting [23] models.

Given the exponential growth in the number of SARS-CoV-2 infections and the pressure in the health care systems, most countries in the world have implemented non-pharmaceutical interventions (NPIs) during the current coronavirus pandemic, designed to reduce human mobility and limit human interactions to contain the spread of the virus. These NPIs range from closing schools and 
non-essential workplaces to requiring citizens to wear masks and limiting national and/or international travel. How to model the impact that the applied NPIs have on the progression of the pandemic is a non-trivial task, particularly for traditional meta-population approaches. Moreover, the social and economic costs of applying NPIs for a sustained period of time has led to the largest global recession in history, with more than a third of the global population under confinement during the first wave of the pandemic in March - April of 2020. The global GDP shrunk by nearly 22 trillion of US dollars as of January 2021, according to the IMF'. Beyond the economic cost, the social cost of the pandemic is also staggering, preventing children and teenagers from attending schools, cancelling cultural activities and forbidding people to visit their friends or relatives.

In view of these challenges, the XPRIZE foundation organized in November of 2020 a global competition called the 500K XPRIZE Pandemic Response Challenge sponsored by Cognizant [1]. This four-month challenge focused on the development of data-driven AI systems to predict COVID-19 infection rates and prescribe Non-pharmaceutical Intervention Plans that governments and communities could implement to minimize harm when reopening their economies.

In this paper, we describe the predictor and prescriptor models developed by ValenciaIA4COVID, the winning team of the competition. The paper is organized as follows: Section 2 provides an overview of the most relevant related work. The data used in the competition is described in Section 3. The predictor and the prescriptor models are presented in Section 4 and 5 , respectively, followed by the experimental results in Section 6 The main conclusions of our work and our future lines of research are outlined in Section 7 .

\section{Related work}

We built a COVID-19 infections predictor based on Long Short Term Memory (LSTM) networks [13. Here, we briefly provide an overview of the approaches that are the most similar to ours, i.e. based on recurrent neural networks. Comparative analyses with other methods can be found in e.g. 25.

Chatterjee et al. 7] applied stacked, bidirectional LSTMs and compared them with multilayer LSTMs. They obtained good accuracy in the prediction of the total number of cases and deaths in the world. Moreover, they did not find any statistical correlation between COVID-19 cases and temperature, sunshine, and precipitation, showing that the number of infections mostly depends on the behavior and density of the population. In [8, LSTMs were used to predict the evolution of the pandemic in Canada and compared it with the USA, Spain, and Italy. Prompt interventions were found to have a strong impact in minimizing the total number of infections, though the accuracy of their predictions was good only for a relatively short time period. Other examples of early works explored using LSTMs to predict COVID-19 cases and the effect of NPIs in India [3] and

1 https://www.dw.com/en/coronavirus-global-gdp-to-sink-by-22-trillion-over-covid-says-imf/a56349323 
Iran [4, with accurate results within a prediction interval of one week up to a month.

Clustering algorithms have been used to improve the models' performance. In 19 the authors use an LSTM to predict cases in different states of Brazil. First, they cluster nations by their temporal series of infections and then assign each Brazilian state to the closest cluster. Global COVID-19 case data was also used in [14 to cluster countries according to their outcomes.

To the best of our knowledge, our work is the first to propose a bank of LSTMs to predict the evolution of the coronavirus pandemic in 236 countries and regions in the world, with good prediction results over a long time period (up to 180 days) and taking into consideration the NPIs applied in each country/region.

Regarding the prescriptor part of our work, there are very few related references. In 24] a multi-objective genetic algorithm was used to find optimal policies using data from Wuhan. Sameni presents an approach to find a balance between interventions and the number of cases with a core compartmental model. This approach requires evaluating the impact of the policy on the evolution of the disease 22]. Several works evaluated the effectiveness of NPIs: see 2120] for studies in Italy, Taiwan and Malaysia or [106] for recent studies in Europe. Finally, Miikkulainen et al. propose a neuroevolution approach to identify a Pareto-optimal set of NPIs [17, that was recommended during the Challenge.

\section{Data}

The coronavirus is the first global pandemic for which there is extensive data captured and shared on a daily basis for most countries and regions in the world. The Challenge leveraged publicly available official COVID-19 case data together with the Oxford COVID-19 Government Response Tracker data set ${ }^{2}$ as the main data sources to be used during the competition [11. This data set provides information for 186 countries and state/region-level data for the US, UK, Canada, and Brazil. The Challenge considered 182 countrie $^{3}$, the 50 US states and the 4 regions in the UK, yielding a total of 236 countries or regions. In the rest of the paper, we will use GEO to denote the countries/regions.

The available data sources can be split into case-related data, i.e. number of daily confirmed COVID-19 cases, and action or NPI-related data, i.e. the NPIs and their level of activation each day for each GEO. In the Challenge, we considered 12 NPIs of two types: confinement-based and public health-based, that are summarized with all their possible levels of activation in Table 1.

\section{Predictors of COVID-19 cases}

This part of the Challenge required building a predictor of the number of confirmed COVID-19 cases in the 236 GEOs for up to 180 days into the future, and

\footnotetext{
2 https://www.bsg.ox.ac.uk/research/research-projects/coronavirus-government-response-tracker
}

3 Tonga, Malta, Turkmenistan and Virgin Islands- were not considered due to lack of reliable data. 
Table 1. NPIs considered in the Challenge and their possible activation values. The predictor is trained with confinement interventions ( $\mathrm{C} 1$ to $\mathrm{C} 8$ ). Both confinement and public health interventions ( $\mathrm{H} 1$ to $\mathrm{H} 3$ and $\mathrm{H} 6$ ) are considered in the prescriptor.

\begin{tabular}{|l|c|l|c|}
\hline NPI name & Values & NPI name & Values \\
\hline C1. School closing & {$[0,1,2,3]$} & C7. Internal movement restrictions & {$[0,1,2]$} \\
\hline C2. Workplace closing & {$[0,1,2,3]$} & C8. International travel controls & {$[0,1,2,3]$} \\
\hline C3. Cancel public events & {$[0,1,2]$} & H1. Public information campaigns & {$[0,1,2]$} \\
\hline C4. Restrictions on gatherings & {$[0,1,2,3]$} & H2. Testing policy & {$[0,1,2,3]$} \\
\hline C5. Close public transport & {$[0,1,2]$} & H3. Contact tracing & {$[0,1,2]$} \\
\hline C6. Stay at home requirements & {$[0,1,2,3]$} & H6. Facial coverings & {$[0,1,2,3,4]$} \\
\hline
\end{tabular}

considering the different NPIs implemented in each GEO. Evidently, the NPIs should impact the transmission of the disease and hence the number of cases. Next, we summarize our notation, followed by a description of our deep learning-based predictive model.

\subsection{Notation}

In the following, we will use the following terms and notation:

1. GEO: We denote as GEO a country or a region (e.g. California). We use the index $j$ to refer to each GEO.

2. Population $\left(P^{j}\right): P^{j}$ denotes the total population of GEO $j$. We assume that each GEO's population is constant during the entire period of time.

3. NewCases $\left(X_{n}^{j}\right)$ : The daily number of new cases on day $n$ and GEO $j$ is denoted by $X_{n}^{j}$. The first day considered is March, 11th 2020.

4. ConfirmedCases $\left(Y_{n}^{j}\right)$ : The cumulative number of confirmed cases up to day $n$ in GEO $j$ is given by $Y_{n}^{j}=\sum_{i=1}^{n} X_{i}^{j}$.

5. SmoothedNewCases $\left(Z_{n}^{j}\right)$ : We compute the average number of new cases between days $n-K+1$ and $n$ in GEO $j$ as $Z_{n}^{j}=\frac{1}{K} \sum_{i=0}^{K-1} X_{n-i}^{j}$. This prevents noise due to different imputation policies (some GEOs do not report cases on weekends, while others do). We use $K=7$ to smooth over one week.

6. CaseRatio $\left(C_{n}^{j}\right)$ : The ratio of cases between two consecutive days is denoted by $C_{n}^{j}=Z_{n}^{j} / Z_{n-1}^{j}$. It indicates the growth/decrease in the number of cases.

7. Susceptible Population $\left(S_{n}^{j}\right)$ : The number of susceptible individuals to be infected with coronavirus on day $n$ and for GEO $j$ is denoted by $S_{n}^{j}$.

8. ScaledCaseRatio $\left(R_{n}^{j}\right)$ : It is the CaseRatio $C_{n}^{j}$ divided by the proportion of susceptible individuals in GEO $j, R_{n}^{j}=C_{n}^{j} \frac{P^{j}}{S_{n}^{j}}$. It captures the effects of a finite population, as it depends on proportion of susceptible individuals in GEO $j$.

9. Action $\left(A_{n}^{j}\right)$ : The vector with the applied NPIs in GEO $j$ on day $n$.

10. Stringency of $A_{n}^{j}\left(\operatorname{Str}_{A_{n}}^{j}\right)$ : The stringency of an NPI applied in GEO $j$ on day $n$ is given by $\operatorname{Str}_{A_{n}}^{j}=\sum_{i=C 1}^{H 6} a_{n}^{j}(i) \cdot \operatorname{Cost}^{j}(i)$, where $\operatorname{Cost}^{j}$ is the cost vector of each of the 12 different types of NPIs ([C1...C8,H1,H2,H3,H6]) in GEO $j$.

11. Intervention Policy (IP): The sequence of daily 12-dimensional NPI or 
action vectors applied over a time period $T$.

12. Stringency of an Intervention Policy: The sum of the stringencies of the NPIs or actions $A_{n}^{j}$ applied each day $n$ over the time period $T$.

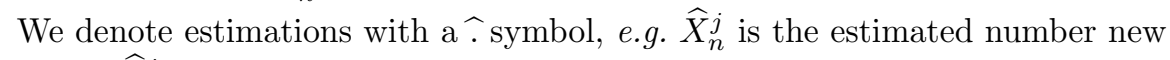
cases and $\widehat{R}_{n}^{j}$ the estimated scaled case ratio, both for GEO $j$ and day $n$.

\subsection{SIR Epidemiological Model}

The predictors model the dynamics of the epidemics in each GEO $j$ using an underlying basic SIR compartmental meta-population model [2]. In this model, the population is divided into three different states: S (Susceptible), Z (Infected), and $\mathrm{D}$ (Removed, due to recovery or death). The dynamics of such an SIR model is included in the S.M. The evolution of the number of infected individuals is given by $\frac{d Z^{j}}{d t}=\beta \frac{S^{j}}{P_{j}} Z^{j}-\mu Z^{j}$, where $\beta$ is the infection rate which controls the probability of transition between the $S$ and $Z$; and $\mu$ is the recovery or removal rate, controlling the probability of transition between the $Z$ and $D$ states. When discretizing $\frac{d Z^{j}}{d t}$ for two consecutive days, we obtain

$$
Z_{n}^{j}=Z_{n-1}^{j}+\beta \frac{S_{n-1}^{j}}{P_{j}} Z_{n-1}^{j}-\mu Z_{n-1}^{j}=\left(1+\beta \frac{S_{n-1}^{j}}{P_{j}}-\mu\right) Z_{n-1}^{j} .
$$

which yields

$$
R_{n}^{j}=\frac{(1-\mu) P_{j}}{S_{n}^{j}}+\beta=\frac{Z_{n}^{j}}{Z_{n-1}^{j}} \frac{P^{j}}{S_{n}^{j}} .
$$

This equation links $R_{n}^{j}$ with the parameters of the SIR model. The larger the $R_{n}^{j}$, the larger $\frac{Z_{n}^{j}}{Z_{n-1}^{j}}$ and hence the larger the growth in the number of cases. Given that $\mu$ is constant in (2), the larger the infection rate $\beta$, the larger the $R_{n}^{j}$. Moreover, the infection rate and thus $R_{n}^{j}$ depend on the applied NPIs.

If we predict $\widehat{R}_{n}^{j}$, we can estimate the number of cases for day $n$ at GEO $j$ :

$$
\widehat{X}_{n}^{j}=\left(\widehat{R}_{n}^{j} \frac{S_{n-1}^{j}}{P_{j}}-1\right) K Z_{n-1}^{j}+X_{n-K}^{j} .
$$

where $K=7$ is the size of the temporal window used to compute $Z_{n}$. As previously explained, $X_{n-K}$ is the reported new cases for day $n-K ; \widehat{R}_{n}^{j}$ is the predicted $R_{n}^{j} ; P^{j}$ is the population of GEO $j$; and $Z_{n-1}^{j}$ is the cumulative number of cases averaged over $K$ days for day $n-1$ in GEO $j$.

Thus, the goal of the predictors is to estimate $\widehat{R}_{n}^{j}$ given the data up to day $n-1$. Since $R_{n}^{j}$ depends on the transmission rate and the dependency of the transmission rate on the NPIs, the predictors consider the number of COVID-19 infections (context) and the applied NPIs (actions) each day in each GEO. 


\subsection{Baseline or standard predictor}

The baseline or standard predictor was provided by the Challenge organizers 17 . It consists of two parallel LSTMs, one to model the context - given by the $R_{n}^{j}$ - and the other to model the actions $\left(A_{n}^{j}\right)$ applied on day $n$ in GEO $j$. Figure 1 (left) depicts the architecture of this baseline model. It uses the context and action data to get predictions separately, joining both outputs via a lambda merge layer.
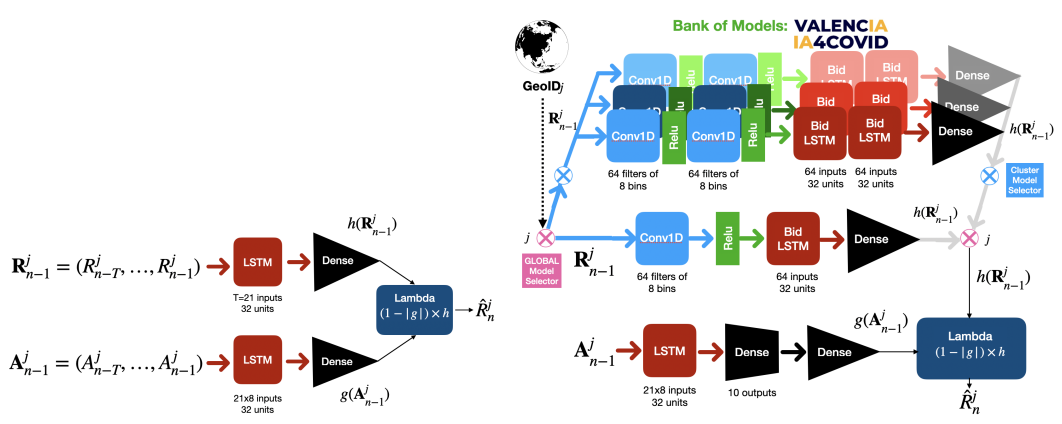

Fig. 1. Left: Baseline LSTM-based predictor; Right: ValenciaIA4COVID predictor.

The lambda layer combines the output of the context LSTM $h$ (top) and the output of the action LSTM $g$ (down), represented in Figure 1. The input to the LSTM $h$ is the vector of values of $R_{n}$ in the previous $T$ days in GEO $j$, namely $\mathbf{R}_{n-1}^{j}=\left(R_{n-T}^{j}, \ldots, R_{n-1}^{j}\right)$. The input to the LSTM $g$ is the matrix of 12-dimensional NPIs (actions) taken during the previous $T$ days in GEO $j$, namely $\mathbf{A}_{n-1}^{j}=\left(A_{n-T}^{j}, \ldots, A_{n-1}^{j}\right)$.

In our experiments we set $T=21$, similarly to [17. Such time window mitigates the noise due to how different GEOs report cases (e.g. Spain does not report confirmed cases during the weekends and holidays, France reports just four days per week, etc.). Moreover, this temporal granularity enables the model to consider the average period of 12-15 days between being exposed to the coronavirus, being detected and tested as a new confirmed case [15].

The output of the lambda layer for day $n$ is the predicted $\widehat{R}_{n}^{j}$ given by

$$
\widehat{R}_{n}^{j}=f\left(\mathbf{A}_{n-1}^{j}, \mathbf{R}_{n-1}^{j}\right)=\left(1-g\left(\mathbf{A}_{n-1}^{j}\right)\right) h\left(\mathbf{R}_{n-1}^{j}\right)
$$

with $g\left(\mathbf{A}_{n-1}^{j}\right) \in[0,1]$ and $h\left(\mathbf{R}_{n-1}^{j}\right) \geq 0$. More details about the baseline model can be found in [17]. Note that when making predictions into the future, the $R_{n-i}^{j}$ values in the vector $\mathbf{R}_{n}^{j}$ are replaced by the estimations provided by the predictor, namely $\widehat{R}_{n-i}^{j}$, for $n-i>$ current $_{\mathrm{d}}$ ay, $i=1, \ldots, T$. 


\subsection{ValenciaIA4COVID (V4C) predictor}

Similarly to the baseline predictor, we implemented an architecture with 2 LSTMbased branches: a context branch, where we modeled the $R_{n}$ time series and an action branch, where we modeled the time series of the eight confinement-based ([C1...C8]) Non-pharmaceutical Interventions. While we did not consider public health-based NPIs, we improved the baseline predictor in several ways. We denote this improved model as the ValenciaIA4COVID or V4C predictor.

\subsubsection{Context branch}

We identified large variability in the time series of confirmed COVID-19 cases depending on the GEO, which made it difficult for a single LSTM context model to perform well everywhere. More precisely, the analysis of the weights of a single model trained on all the data showed that the LSTM matrices were full rank. Hence, we opted for a bank of LSTM context models, shown in Figure 1 (right).

Bank of context models We created the bank as follows: First, we clustered the GEOs via a K-means algorithm applied to the time series of reported number of COVID-19 cases per 100K inhabitants. We optimized the number of clusters using the Elbow method, obtaining 15 different clusters shown in the S.M.

Next, we trained a reference LSTM model with data from the 20 most-affected GEOs and 15 different cluster LSTM models using data from all the GEOs in each of the 15 clusters. In our experiments, we set March 11th, 2020 as the starting date for training the models. We then evaluated the reference and all the cluster models on our testing data for all the GEOs. Our testing period started on Nov. 1st for long-term evaluation and Dec. 1st for short-term evaluation, ending on Dec. 21st, 2020. We automatically selected the model with the lowest MAE per 100K inhabitants in each GEO, applying Occam's razor principle to minimize the number of models in our bank. Thus, we favored the reference model when it obtained a similar performance to the best of the cluster models. As a result of this process, we selected nine models: the reference model, applied in 135 GEOs; and eight cluster models applied in the remaining GEOs. A visualization of the cluster and model assignments can be found here 4 .

LSTMs Architecture. In the context branch $(h)$ we implemented two different LSTM-based architectures, as depicted in Figure 1 (right): one for the reference model and the other for each of the eight cluster models. The reference model includes a convolutional layer with ReLu activation function and a bidirectional LSTM followed by a dense layer. Each convolutional layer has 64 filters of size 8 . This reference model empirically generalized well for 135 GEOs.

The cluster models consist of a stacked version of the architecture of the reference model, with two convolutional layers and two stacked bidirectional LSTMs. Each convolutional layer also has 64 filters of size 8 with ReLu as the activation function and add a final dense layer. After the double $1 D$ convolution spans the characterization of the input sequence, the first LSTM encodes such a characterization in states of 64 dimensions (bidirectional) and feeds into the

\footnotetext{
4 https://tinyurl.com/cjstz4yc
} 
second LSTM, whose units can now operate at a different time scale. This added complexity enabled the models to perform well in the GEOs where the reference model did not. After model selection, we obtained a bank of eight different cluster models.

\subsubsection{Action branch}

We used an LSTM followed by two dense layers to smooth the output and hence better capture non-linearities. Similarly to [17, we used a sigmoid activation function to guarantee that the action layer's output to be in [0,1]. Since increasing the activation or stringency of an NPI should not decrease its effectiveness, $g$ is constrained to satisfy the condition: if $\min \left(A-A^{\prime}\right) \geq 0 \longrightarrow g(A) \geq g\left(A^{\prime}\right)$. This constraint is enforced by setting all trainable parameters of $g$ to be non-negative (absolute value) after each parameter update. Note that convolution here is not considered in order to keep the raw NPI constraints. The V4C predictor only considers the confinement NPIs, so each $A_{n}^{j}$ is an 8-dimensional vector with the level of activation of the eight confinement NPIs (see Table 1).

\subsubsection{Merge function}

The two branches use the data from the last 21 days that are combined into a final dense layer to get the predicted $\widehat{R}_{n}$. The outputs of each branch ( $h$ and g) are merged by the lambda function defined in (4). Thus, the predicted $\widehat{R}_{n}$ provided by the context branch is modified by the output from the action branch. The stricter the NPIs, the larger the output from the action layer, thus reducing the context layer's output. Finally, once the model gives the predicted $\widehat{R}_{n}$, the predicted number of new infections for day $n, \widehat{X}_{n}$, is obtained using (3).

\section{Prescriptor of Intervention Policies}

The final phase of the XPRIZE competition required building a prescriptor which would recommend for each GEO and for any period of time, up to 10 different Intervention Policies (IP) with the best balance between their economic/social cost and the resulting number of COVID-19 cases.

Thus, it entailed solving a two-objective optimization problem by identifying the set of solutions that would be on the Pareto front [5]16[17. On the one hand, there is the stringency of a certain IP which captured the sum of the costs of implementing such a policy. On the other hand, there is the number of COVID-19 cases per $100 \mathrm{~K}$ inhabitants which would result from applying such IP. Given that this is a hypothetical scenario, the number of COVID-19 infections under the IPs was estimated by the baseline or standard predictor provided by the XPRIZE Challenge organizers. All the teams used the same predictor to enable the judges to compare the prescriptors from different teams properly.

Our goal in the Prescription phase of the competition was to develop an interpretable, data-driven and flexible prescription framework that would be usable by non machine-learning experts, such as citizens and policymakers in the Valencian Government. Our design principles were therefore driven by developing explainable and transparent models. The Challenge entailed finding the set of Pareto-optimal IPs with the best trade-off between their economic/social 


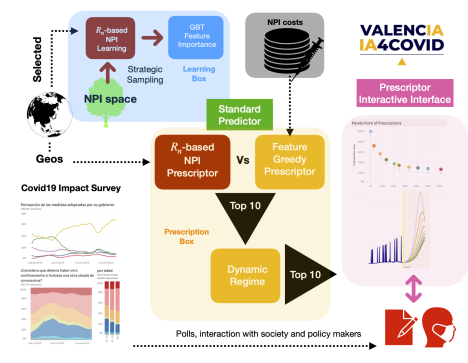

Fig. 2. V4C Prescriptor. The (offline) learning box (in blue) infers the convergence $\widehat{R}_{n}$ for the sampled NPIs, and the Gradient Boosted Trees identify the feature importance. The prescriptor relies on the standard XPRIZE predictor. The first set of NPIs is obtained by the NPI- $\widehat{R}_{n}$ mapping; the second set, using a feature importance-based greedy algorithm. These two sets compete and up to 10 non-dominated IPs are selected.

costs and their associated number of resulting COVID-19 cases. An intervention policy $I P_{1}$ dominates another intervention policy $I P_{2}$ if the stringency $\left(I P_{1}\right) \leq$ stringency $\left(I P_{2}\right)$ and the resulting number of COVID-19 cases under $I P_{1}<$ than under $I P_{2}$. The goal was to find up to 10 IPs for each GEO, for any time period and any costs that would dominate the rest of possible IPs. As in the case of our predictor, we decided to combine complementary approaches to have a more robust solution, shown in Figure 2.

\subsection{Modeling the NPI - COVID-19 cases space}

Before building the prescriptor, we performed an exploratory data analysis of the problem space. Our goal was to shed light on the relationship between the NPIs and the resulting number of COVID-19 cases. Considering all the possible values of each dimension of the NPI or action vector, there are 7,776,000 possible combinations of NPI vectors that could be applied at each time step.

Each NPI vector, when applied for a minimum amount of time, would lead to a reduction or increase in the number of COVID-19 cases in the GEO where it is applied (see Equation 4). To better understand the impact that different NPI vectors have on the number of COVID-19 cases, we ran numerous experiments where we called the predictor with different values of the NPI vector over varying time periods of 30 to 90 days and on a sample of 21 representative GEOs from different continent5 5 namely: United States, Brazil, India, Mexico, Italy, China, United Kingdom, France, England, Russia, Iran, Spain, Argentina, Colombia, New York State, Peru, Germany, Poland, South Africa, Texas and California. For each case, we obtained the resulting $\widehat{R}_{n}$ estimated by the predictor.

In our experiments, we observed that the same NPI vector would lead to the same convergence $\widehat{R}_{n}$ in all the GEOs and over any time period provided that the NPI was applied for long enough (see a justification in the S.M.).

\footnotetext{
${ }^{5}$ We selected amongst the most affected countries and regions across the globe.
} 
Moreover, we found that the convergence time of $\widehat{R}_{n}$ is inversely proportional to its value. As per Eq. (2), note that the larger the $\widehat{R}_{n}$, the larger the number of resulting COVID-19 cases. We refer to this finding as the $R_{n}$ synchronization principle. Moreover, all countries underwent a transitory period of $\approx 21$ days since the application of a certain NPI before their $\widehat{R}_{n}$ started converging towards its convergence value. Figure 3 illustrates the convergence of the $\widehat{R}_{n}$ for two different NPI vectors in the 21 selected GEOs.

\subsection{Prescriptors}

\subsubsection{Prescriptor Method 1: $R_{n}$-based NPI selection}

Based on the $R_{n}$ synchronization principle, one could easily obtain the Paretooptimal front of intervention policies if the mapping between the 7.8 million of possible combinations of the NPI vector and their associated convergence $\widehat{R}_{n}$ were to be known. Unfortunately, generating such a mapping was not feasible in the time frame provided by the Challenge as it would require making millions of calls to the predict function. Hence, we opted for computing a sample of such a matrix (whose distribution is shown in the S.M.), obtained as (1) all the NPI vectors with stringencies [0 to 6] and [28 to 34]; (2) all NPI vectors with one and two non-zero entries; and (3) a random sample of 10,000 NPIs.

For each NPI in the sample, we computed the convergence $\widehat{R}_{n}$, and the resulting total number of COVID-19 cases in 20 and 60 days.

Using this NPI- $\widehat{R}_{n}$ matrix, we trained state-of-the-art machine-learning models to predict the $\widehat{R}_{n}$ for any given NPI vector. The best performing and explainable model were Gradient Boosted Trees, which obtained a MAE on the test set of 0.0003. While such MAE was still too large for us to be able to fill-in all the missing elements in the NPI- $\widehat{R}_{n}$ matrix, we carried out a feature importance analysis and discovered that the $\mathrm{C} 2, \mathrm{C} 1, \mathrm{H} 2, \mathrm{C} 4$ and $\mathrm{C} 5$ interventions are, in this order, the most important to predict their associated $\widehat{R}_{n}$ and hence the resulting number of COVID-19 cases (see S.M. for details).

Thus, we also included in our NPI- $\widehat{R}_{n}$ matrix all the NPI vectors with nonzero values in their $\mathrm{C} 1, \mathrm{C} 2, \mathrm{C} 4, \mathrm{C} 5$ and $\mathrm{H} 2$ interventions and zero in the rest of the dimensions. This led to a total of 54,652 NPI vectors.

As a result, we generated a matrix with the mapping between these different NPI vectors, their associated stringencies (at cost 1), the number of cases that they would lead to at 20 days and at 60 days, and their convergence $\widehat{R}_{n}$. We carried out all computations on the sample of 21 previously listed GEOs.

At run time, given an input cost vector, the prescriptor computes the stringency of each row in the NPI- $\widehat{R}_{n}$ matrix and identifies the NPI combinations that are on the Pareto front by selecting those that lead to the best trade-off between their stringencies, their associated number of cases at 20 and 60 days and their convergence $\widehat{R}_{n}$. More details are included in the S.M.

\subsubsection{Prescriptor method 2: Feature greedy NPI selection}

As per the feature importance analysis described above and given a cost vector, we developed a greedy NPI prescriptor as follows: each dimension of the NPI 

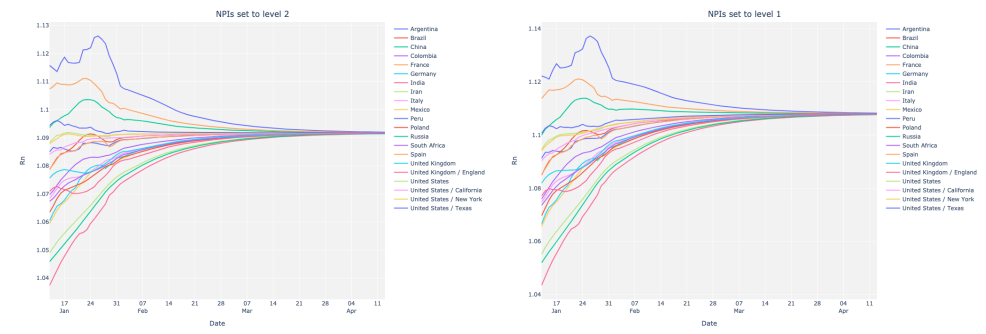

Fig. 3. $\hat{R_{n}}$ convergence for two different NPI vectors on 21 representative GEOs.

vector is ranked by its priority, computed as its feature importance divided by its cost. This prescriptor consists of a greedy algorithm that consecutively activates to its maximum value each NPI dimension by order of its priority. This method is related to the greedy strategies developed to solve the knapsack problem 6 5.2.3.

\section{Prescriptor combination}

Each of the methods above provides a set of NPI recommendations for each GEO for each day. From such a set, we select the 10 best NPIs that satisfy the following criteria: (1) they are not dominated by any other NPI; and (2) they contribute to having a diverse set of NPIs that cover the full range of possible stringency values. Additional details are included in the S.M.

\subsection{Intervention policy definition}

Finally, the prescriptor needs to provide a set of up to 10 Intervention Policies, i.e. dynamic regimes of applying the selected NPIs over the time period of interest. To do so, we compute all possible combinations of subsequently applying the selected NPIs in chunks of minimum 14 days (to enable the NPIs to act) and identify the Pareto-front set of combinations that would yield the optimal trade-off between stringency and number of cases. The total number of chunks is dynamically determined. From this set of combinations, we again select the 10 that (1) are not dominated by any other policy; (2) contribute to having a diverse set of policies along the stringency axis and (3) minimize the changes in NPIs, as every NPI change has a social cost from a practical perspective.

Two screenshots of the interactive visualization that we developed so policymakers could easily compare the prescribed IPs are shown in the S.M. and can be found here?

\section{$6 \quad$ Experimental results}

In this Section, we report the results of quantitatively evaluating our predictor both in short and long-term prediction scenarios and qualitatively assessing

6 See https://en.wikipedia.org/wiki/Knapsack_problem.

7 https://public.tableau.com/app/profile/kristina.p8284/viz/PrescriptionsWeb/Visualize 
the performance of our predictor and prescriptor in hypothetical scenarios. Our source code is publicly available 8 .

\subsection{Predictor}

We evaluated the predictive performance of our COVID-19 cases predictor and compared it to the baseline model under different scenarios. We computed both the Mean Absolute Error (MAE) of the estimated number of COVID-19 cases per $100 \mathrm{~K}$ inhabitants for each GEO in the Challenge and the Mean Rank of our model when compared to the baseline model.

All the models were trained with data from the Oxford COVID-19 Government Response Tracker dataset, from March 11th to December 17th 2020, for the 20 most affected countries in terms of confirmed cases.
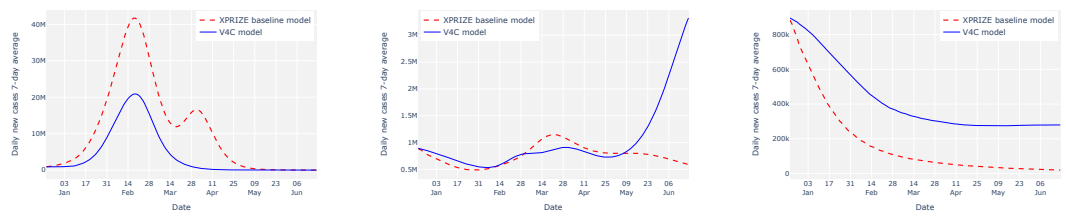

Fig. 4. Smoothed predicted daily new cases worldwide (7-day average) for three different future scenarios based on different values of the NPI vector: zero (left), frozen (center) and maximal (right) NPIs applied. Note how without any NPIs there is a large wave of infections, which is avoided when the NPIs set to their maximal values.

As the consistency of the model is an important characteristic to assess, we evaluated the models both in short-term and long-term predictions. Short-term evaluations consisted in generating predictions for 3 weeks ahead into the future for the time period between Dec 1st and Dec 21st, 2020. Long-term evaluations were two-fold: First, with historic data, we tested the predictions between Nov 1st and Dec 21st, 2020; Second, we ran the predictors under three different 180-day prediction scenarios: (i) a scenario where the NPIs were frozen as of their values in Dec 21st 2020; (ii) a scenario with all NPIs in all GEOs were set to their maximum levels; and (iii) a scenario where all NPIs in all GEOs were set to 0. The behavior of our model under these three conditions made intuitive sense, as depicted in Figure 4.

Table 2 displays the MAE per 100K inhabitants and the Mean Rank of the proposed model when compared to the baseline model provided by the XPRIZE organizers. We also include the results of only using our reference context model without the clusters. As seen on the Table, our model outperforms the baseline model in all evaluation scenarios in terms of MAE and Mean Rank. Moreover,

\footnotetext{
8 https://github.com/malozano/valencia-ia4covid-xprize
} 
during the predictor evaluation phase of the XPRIZE Challenge, our predictor ranked third in the world in Mean Rank amongst all the teams, first in Mean Rank in Asian and in European GEOs. As per our collaboration with the President

Table 2. Predictor results in short and long-term evaluations in the 236 GEOS.

\begin{tabular}{|l|c|c|c|c|}
\hline \multirow{2}{*}{ Predictor } & \multicolumn{2}{|c|}{ Short-term } & \multicolumn{2}{c|}{ Long-term } \\
\cline { 2 - 5 } & MAE & Mean Rank & MAE & Mean Rank \\
\hline XPRIZE LSTM baseline & 157.924142 & 2.106383 & 935.340780 & 2.297872 \\
V4C (w/o clusters) & 138.208982 & 2.144681 & 825.375377 & $\mathbf{1 . 8 3 4 0 4 3}$ \\
V4C with clusters & $\mathbf{1 2 6 . 3 3 1 2 1 6}$ & $\mathbf{1 . 7 4 8 9 3 6}$ & $\mathbf{8 0 3 . 5 8 7 3 8 1}$ & 1.868085 \\
\hline
\end{tabular}

of the Valencian Government in Spain, we were able to share the predictions of our predictor during the 3rd wave of the COVID-19 pandemic that started right after Christmas of 2020. Figure 5 shows the predictions of our model (blue) when compared to the baseline predictor (red) and the ground truth (yellow). As seen in the Figure, our predictor was very accurate in predicting the evolution of the pandemic while taking into account the different NPIs that were implemented at the time. It provided valuable input to the Government in their decision-making.

\subsection{Speed and Resource Use}

In terms of training, we used an Intel Core i7 with $256 \mathrm{~Gb}$ RAM and GPU. The training time of the reference model with 20 trials was 108 minutes and of the cluster models ranged between 24 minutes (largest cluster with 106 GEOs) and 44 seconds (smallest cluster with 2 GEOs).

We carried out our prediction experiments on an Intel Core i7, 4 cores, 2,7 Ghz, 16GB 2133MHz LPDDR3. Table 3 (Top) summarizes the times needed to
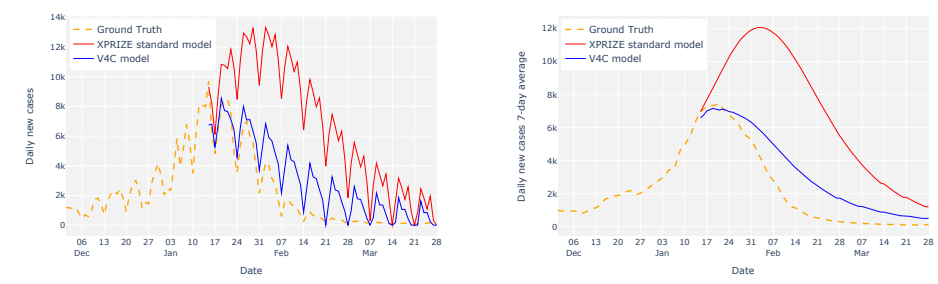

Fig. 5. Predictions vs ground truth for the Valencian region (Spain) during the third wave: daily new cases (left) and smoothed daily new cases (right).

produce a prediction for all the GEOs by the baseline model and our proposed 
Table 3. Top: Total time needed to generate predictions for all the GEOs. Bottom: Prescriptor results: \# of dominating / \# of dominated prescriptions for 5-day (from Aug 1st to Aug 5th, 2020), 31-day (from Jan 1st to Jan 31st, 2021) and 90-day (from Jan 1st to Mar 31st, 2021) time periods.

\begin{tabular}{|c|c|c|c|}
\hline & \multicolumn{3}{|c|}{ Window size of prediction } \\
\hline Predictor & 31-days & $\mathbf{6 1 - d a y s}$ & $\mathbf{1 8 0}$-days \\
\hline Baseline & 212 seconds & 409 seconds & 1,092 seconds \\
\hline V4C & 417 seconds & 597 seconds & 1,239 seconds \\
\hline \hline Prescriptor & $\mathbf{3 1 - d a y s}$ & $\mathbf{6 1 - d a y s}$ & $\mathbf{1 8 0 - d a y s}$ \\
\hline Greedy & $127 / 1814$ & $130 / 1829$ & $163 / 1839$ \\
\hline Feature greedy & $921 / 114$ & $930 / 117$ & $\mathbf{9 8 6} / 163$ \\
\hline V4C prescriptor & $\mathbf{9 2 7} / \mathbf{4 7}$ & $\mathbf{9 3 4} / \mathbf{4 8}$ & $\mathbf{9 8 6} / \mathbf{1 3 7}$ \\
\hline
\end{tabular}

model for three different sizes of the prediction period. As seen on the Table, the computation needs of our model were well below the maximum time allowed in the XPRIZE competition (60 minutes). We favored simplicity in our design and aimed to minimize the energy consumption to be as planet-friendly as possible.

\subsection{Prescriptor}

Given the hypothetical nature of the prescriptor, we were not able to quantitatively evaluate its performance against ground truth. However, we did carry out domination tests between the IPs recommended by our model when compared to a greedy algorithm for the 236 GEOs in the Challenge and under both unitary and random costs policies for a time period of 60 days into the future. Figure 6 depicts the recommended IPs by our model (orange and green) when compared to a greedy prescriptor (blue).

Table 3 (bottom) shows the number of times the IPs recommended by our prescriptor dominated and were dominated by the IPs suggested by the greedy approach for all GEOs. Moreover, our prescriptor provided the IP recommendations in under 2 hours for all GEOs in the Challenge, well below the maximum allowed limit of 6 hours.
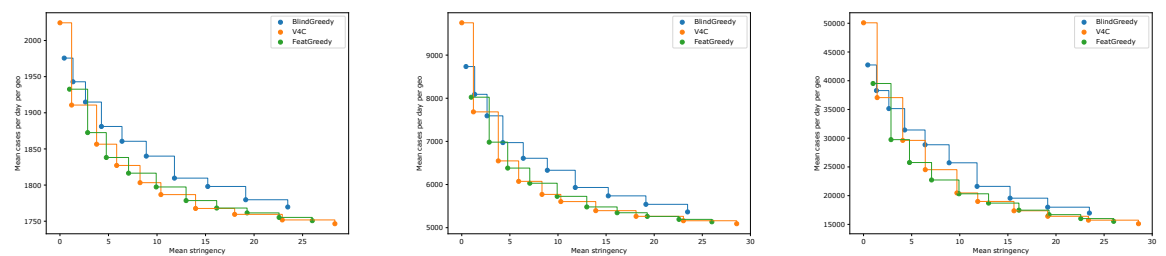

Fig. 6. Number of cases vs stringency obtained from prescriptions generated for 5 days (left), 31 days (center) and 90 days (right). 


\section{Conclusions and Future Work}

In this paper, we have described the models developed by the winning team of the 500K XPRIZE Pandemic Response Challenge. The competition entailed first developing a model to predict the number of COVID-19 cases in 236 countries/regions in the world, for up to 180 days into the future and considering the Non-pharmaceutical Interventions deployed in each country/region. In this phase, we developed an LSTM-based bank of models which outperformed the baseline model provided by the Challenge organizers and yielded the third best Mean Rank amongst all the teams in the competition. The proposed model was successfully used by the President of the Valencian government in Spain during the third wave of COVID-19 infections in December - February 2021.

Next, the teams were asked to develop a prescription model that would recommend up to 10 Intervention Policies (IPs) in each of the 236 GEOs in the world for any time period and costs that would achieve the best trade-off between the total cost of the IP and the resulting number of coronavirus infections. Our winning solution leveraged the $R_{n}$ synchronization principle to provide Pareto-optimal IPs that clearly dominated other approaches.

We believe that this work contributes to the necessary transition to more evidence-driven policy-making, particularly during a pandemic. Future lines of work include developing the intervention prescriptor within the Valencian Government, developing a theoretical proof of the $R_{n}$ synchronization principle and including the impact of vaccinations in our model.

Acknowledgements The authors have been partially supported by grants FONDOS SUPERA COVID-19 Santander-CRUE (CD4COVID19 2020-2021), Fundación BBVA for SARS-CoV-2 research (IA4COVID19 2020-2022) and the Valencian Government. We thank the University of Alicante's Institute for Computer Research for their support with computing resources, co-financed by the European Union and ERDF funds through IDIFEDER/2020/003. MAGM acknowledges funding from MEFP Beatriz Galindo program (BEAGAL18/00203).

\section{References}

1. 500k XPRIZE Pandemic Response Challenge, sponsored by Cognizant, https: //www.xprize.org/challenge/pandemicresponse

2. Allen, L.: Some discrete-time SI, SIR, and SIS epidemic models. Math. Biosci. 124(1), 83-105 (1994)

3. Arora, P., Kumar, H., Panigrahi, B.K.: Prediction and analysis of covid-19 positive cases using deep learning models: A descriptive case study of india. Chaos, Solitons \& Fractals 139, 110017 (2020)

4. Ayyoubzadeh, S., Ayyoubzadeh, S., Zahedi, H., Ahmadi, M., Kalhori, S.: Predicting COVID-19 incidence through analysis of Google trends data in Iran: data mining and deep learning pilot study. JMIR Public Health Surveill. 6(2) (2020)

5. Belakaria, S., Deshwal, A., Doppa, J.: Max-value entropy search for multi-objective bayesian optimization. In: Wallach, H., Larochelle, H., Beygelzimer, A., d'Alché-Buc, F., Fox, E., Garnett, R. (eds.) NeurIPS. vol. 32 (2019) 
6. Brauner, J.M., Mindermann, S., Sharma, M., et al.: Inferring the effectiveness of government interventions against COVID-19. Science 371(6531) (2021)

7. Chatterjee, A., Gerdes, M., Martinez, S.: Statistical explorations and univariate timeseries analysis on COVID-19 datasets to understand the trend of disease spreading and death. Sensors 20(11), 3089 (2020)

8. Chimmula, V.K.R., Zhang, L.: Time series forecasting of covid-19 transmission in canada using LSTM networks. Chaos, Solitons \& Fractals 135, 109864 (2020)

9. Ferguson, N., Cummings, D., Cauchemez, S., et al: Strategies for containing an emerging influenza pandemic in Southeast Asia. Nature 437(7056), 209-214 (2005)

10. Flaxman, S., Mishra, S., Gandy, A., et al: Estimating the effects of nonpharmaceutical interventions on Covid-19 in Europe. Nature 584, 257-261 (2020)

11. Hale, T., Angrist, N., Goldszmidt, R., Kira, B., et al.: A global panel database of pandemic policies (Oxford COVID-19 Government Response Tracker). Nat. Hum. Behav. pp. 1-10 (2021)

12. Hethcote, H.: The mathematics of infectious diseases. SIAM Rev. 42(4), 599-653 (2000)

13. Hochreiter, S., Schmidhuber, J.: Long short-term memory. Neural Comput. 9(8), 1735-1780 (1997)

14. Khan, M., Hossain, A.: Machine learning approaches reveal that the number of tests do not matter to the prediction of global confirmed COVID-19 cases. Front. Artif. Intell. Appl. 3, 90 (2020)

15. Lauer, S., Grantz, K., Bi, Q., Jones, F., et al: The incubation period of coronavirus disease 2019 (COVID-19) from publicly reported confirmed cases: estimation and application. Ann. Intern. Med. 172(9), 577-582 (2020)

16. Lu, Z., Whalen, I., Dhebar, Y., Deb, K., et al.: NSGA-Net: Neural architecture search using multi-objective genetic algorithm (extended abstract). In: Bessiere, C. (ed.) Proc. of the 29th Int. J. Conf. on AI, IJCAI-20. pp. 4750-4754 (2020)

17. Miikkulainen, R., Francon, O., Meyerson, E., Qiu, X., et al.: From prediction to prescription: Evolutionary optimization of nonpharmaceutical interventions in the COVID-19 pandemic. IEEE Trans. Evol. Comput. 25(2), 386-401 (2021)

18. Pastor-Satorras, R., Castellano, C., Van Mieghem, P., Vespignani, A.: Epidemic processes in complex networks. Rev. Mod. Phys. 87, 925-979 (Aug 2015)

19. Pereira, I., Guerin, J., Silva J., A.G., Garcia, G., Piscitelli, P., Miani, A., Distante, C., Gonçalves, L.: Forecasting COVID-19 dynamics in Brazil: a data driven approach. Int. J. Environ. Res. Public Health 17(14), 5115 (2020)

20. Rahman, M., Zaman, N., Asyhari, A., Al-Turjman, F., et al: Data-driven dynamic clustering framework for mitigating the adverse economic impact of COVID-19 lockdown practices. Sustain. Cities Soc. 62, 102372 (2020)

21. Riccardi, A., Gemignani, J., Fernández-Navarro, F., Heffernan, A.: Optimisation of non-pharmaceutical measures in COVID-19 growth via neural networks. IEEE Trans. Emerg. Topics Comput. 5(1), 79-91 (2021)

22. Sameni, R.: Model-based prediction and optimal control of pandemics by nonpharmaceutical interventions. arXiv preprint arXiv:2102.06609 (2021)

23. Tayarani, N., Mohammad, H.: Applications of artificial intelligence in battling against COVID-19: A literature review. Chaos, Solitons \& Fractals p. 110338 (2020)

24. Yousefpour, A., Jahanshahi, H., Bekiros, S.: Optimal policies for control of the novel coronavirus disease outbreak. Chaos, Solitons \& Fractals 136 (2020)

25. Zeroual, A., Harrou, F., Dairi, A., Sun, Y.: Deep learning methods for forecasting COVID-19 time-series data: A comparative study. Chaos, Solitons \& Fractals 140 (2020) 